\title{
The Alpha-Logarithmic Series Distribution of Order $k$ and Some of Its Applications
}

\author{
C. Satheesh Kumar \\ Department of Statistics, University of Kerala, \\ Trivandrum - 695 581, India \\ E-mail:drcsatheeshkumar@gmail.com \\ A. Riyaz \\ Department of Statistics, University of Kerala, \\ Trivandrum - 695 581, India \\ E-mail: riyazstatoyour@gmail.com
}

Received 11 November 2014

Accepted 4 April 2016

\begin{abstract}
Here we develop an order $k$ version of the alpha-logarithmic series distribution of Kumar and Riyaz (South African Statist. J., 2014) through its probability generating function and derive its probability mass function, mean and variance. The parameters of the model are estimated by the method of maximum likelihood and the distribution has been fitted to certain real life data sets. Certain test procedures are considered for testing the significance of the additional parameters of the distribution. In addition, a simulation study is conducted for assessing the performance of the likelihood estimators of each of the parameters of the model.
\end{abstract}

Keywords: Count data models; generalized likelihood ratio test; logarithmic series distribution; maximum likelihood estimation; Markov chain Monte Carlo simulation; probability generating function; Rao's efficient score test.

2000 Mathematics Subject Classification: 62E10, 53C35

\section{Introduction}

The logarithmic series distribution (LSD) was introduced as the limit of a zero-truncated negative binomial distribution by Fisher et al. [4] to investigate the distribution of butterflies in the Malayan Peninsula. The LSD has been used extensively by Williams [28, 29] for fitting ecological data on species of abundance with long tails. An application of the LSD to an inventory control problem in steel industry appears in Williamson and Bretherton [30]. Chatfield et al. [2] utilized the LSD to represent the distribution of number of items of a product purchased by a buyer in a specified time period. The LSD has been found application in several practical situations such as the studies on sampling of quadrants for plant species, the distribution of animal species, population growth, biology, economics, inventory models and marine sciences. For details see Jonson et al. [6]. Due to practical suitability of the LSD in case of data with long tails, several generalized forms of it have been proposed in the literature. For example see Jain and Gupta [5], Kempton [7], Tripathi and Gupta [26, 27], Ong [20], Khang and Ong [8] and Kumar and Riyaz [14, 15]. 
An important drawback of the LSD in certain practical situations is that it excludes the zero observation from its support. To mitigate this drawback, Khatri [9] has considered a distribution namely "the logarithmic-withzeros distribution (LWZD)" through the following probability mass function (pmf), in which $0<\omega, \theta<1$

$$
f(x)= \begin{cases}\omega, & x=0 \\ \frac{(1-\omega) \theta^{x}}{-x \ln (1-\theta)}, & x=1,2,3, \ldots\end{cases}
$$

Some aspects of the LWZD are also detailed in Johnson et al. (6, pp. 355). Kumar and Riyaz [16] considered a modified form of the LSD with a non-negative support, namely "the alpha- logarithmic series distribution (ALSD)" through the following pmf in which $A=[-\ln (1-\theta-\alpha)]^{-1}, \theta>0$ and $\alpha \geq 0$ such that $\theta+\alpha<1$.

$$
g(x)=\left\{\begin{array}{lc}
-A \ln (1-\alpha), & x=0 \\
A(1-\alpha)^{-x} \frac{\theta^{x}}{x}, & x=1,2,3, \ldots
\end{array}\right.
$$

The probability generating function (pgf) of the ALSD (1.2) with pmf is the following.

$$
G(t)=-A \ln (1-\theta t-\alpha)
$$

Recently there has been renewed interest in the study of discrete distributions of order $k$ in the literature. For example see Kumar [10, 11], Kumar and Shibu [18] or Kumar and Nair [12, 13]. For a detailed account of order $k$ distributions and their applications see section 10.7 of Johnson et al. [6]. Philippou [22] introduced and studied a negative binomial distribution of order $k$, which is also known as the type I waiting time distribution of order $k$. As a limiting form of the left-truncated version of this negative binomial distribution of order $k$, a logarithmic distribution of order $k$ is proposed in the literature as given in page 461 of Johnson et al. [6]. Panaretos and Xekalaki [21] obtained another logarithmic distribution of order $k$ as a limiting form of the gamma-mixed Poisson distribution of order k. Kumar and Riyaz [17] introduced and studied an order $k$ version of the logarithmic series distribution, which they obtained as the limiting case of the zero-truncated cluster negative binomial distribution. All these order $k$ versions of logarithmic distribution do not have nonnegative support. Through the present paper we consider an order $k$ version of the ALSD, which possess a non-negative support and named it as "the alpha-logarithmic series distribution of order $k$ " or in short " the $\operatorname{ALSD}(k)$ ". In section 2 we present the definition of the $\operatorname{ALSD}(k)$ and derive its pmf, mean and variance. In section 3 we estimate the parameters of the $\operatorname{ALSD}(k)$ by the method of maximum likelihood and in section 4 we illustrate its usefulness with the help of certain real life data sets. In section 5 we consider the generalized likelihood ratio test and Rao's efficient score test for testing the significance of the additional parameters of the model and in section 6, we carried out a simulation study for comparing the performance of the estimators obtained by the method of maximum likelihood.

We need the following series representation in the sequel. For any real valued function $Q(r, s)$,

$$
\sum_{s=0}^{\infty} \sum_{r=0}^{\infty} Q(r, s)=\sum_{s=0}^{\infty} \sum_{r=0}^{s} Q(r, s-r),
$$

in which $[a]$ denote the integer part of $a$ and

$$
(1-u)^{-b}=\sum_{r=0}^{\infty}(b)_{r} \frac{u^{r}}{r !},
$$

for any $b>0$ and $|u|<1$. 


\section{Definition and Properties}

In this section first we present the definition of the $\operatorname{ALSD}(k)$ and discuss some of its important properties.

Definition 2.1 A non-negative integer valued random variable $Y$ is said to follow "the alpha- logarithmic series distribution of order $k$ " or in short " the $\operatorname{ALSD}(k)$ " if its $\operatorname{pgf}$ is of the following form, in which $\Lambda=\left[-\ln \left(1-\sum_{j=1}^{k} \theta_{j}-\alpha\right)\right]^{-1}, \theta_{j}>0$ and $\alpha \geq 0$ such that $\sum_{j=1}^{k} \theta_{j}+\alpha<1$.

$$
H(t)=-\Lambda \ln \left(1-\sum_{j=1}^{k} \theta_{j} t^{j}-\alpha\right)
$$

Clearly, when $k=1$ the pgf (2.1) reduces to the pgf of the ALSD of Kumar and Riyaz [16] and when $\alpha=0$ the pgf (2.1) reduces to the pgf of the of the logarithmic series distribution of order $k$ studied by Kumar and Riyaz [17]. The pgf of $\operatorname{ALSD}(k)$ given in (2.1) can also be written as

$$
H(t)=\frac{\left(\sum_{j=1}^{k} \theta_{j} t^{j}+\alpha\right)}{\left(\sum_{j=}^{k} \theta_{j}+\alpha\right)} \frac{{ }_{2} F_{1}\left(1,1 ; 2 ; \sum_{j=1}^{k} \theta_{j} t^{j}+\alpha\right)}{{ }_{2} F_{1}\left(1,1 ; 2 ; \sum_{j=1}^{k} \theta_{j}+\alpha\right)}
$$

where

$$
{ }_{2} F_{1}(a, b ; c ; z)=\sum_{r=0}^{\infty} \frac{(a)_{r}(b)_{r}}{(c)_{r}} \frac{z^{r}}{r !}
$$

is the Gauss hypergeometric function, in which $(d)_{r}=d(d+1) \cdots(d+r-1)$, for $r \geq 1$ and $(d)_{0}=1$, for any $d \in R=(-\infty, \infty)$. For details regarding Gauss hypergeometric function see Mathai and Haubold [19] or Slater [25].

Next we derive the pmf of the $\operatorname{ALSD}(k)$ through the following theorem.

Theorem 2.1 The $\operatorname{pmf} q(y)=P(Y=y)$ of the $\operatorname{ALSD}(k)$ with $\operatorname{pgf}(2.1)$ is the following.

$$
q(y)= \begin{cases}-\Lambda \ln (1-\alpha), & y=0 \\ \Lambda \sum_{J_{y}}(1-\alpha)^{-(1+y)} n ! \frac{\theta_{1}^{y_{1}}}{y_{1} !} \frac{\theta_{2}^{y_{2}}}{y_{2} !} \cdots \frac{\theta_{k}^{y_{k}}}{y_{k} !}, & y=1,2, \ldots,\end{cases}
$$

in which $\sum_{J_{y}}$ denote the summation over all $k$-tuples $\left(y_{1}, y_{2}, \ldots, y_{k}\right)$ of non-negative integers in the set $J_{y}=\left\{\left(y_{1}, y_{2}, \ldots, y_{k}\right): \sum_{j=1}^{k} j y_{j}=y\right\}$ and $n=\sum_{j=1}^{k} y_{j}-1$.

Proof. From (2.1) we have the following.

$$
\begin{aligned}
H(t) & =\sum_{y=0}^{\infty} q(y) t^{y} \\
& =-\Lambda \ln \left(1-\sum_{j=1}^{k} \theta_{j} t^{j}-\alpha\right) .
\end{aligned}
$$


Expand the logarithmic function in (2.6), to get

$$
\begin{aligned}
H(t) & =\Lambda \sum_{n=1}^{\infty} \frac{\left(\sum_{j=1}^{k} \theta_{j} t^{j}+\alpha\right)^{n}}{n} \\
& =\Lambda \sum_{n=0}^{\infty} \frac{\left(\sum_{j=1}^{k} \theta_{j} t^{j}+\alpha\right)^{n+1}}{n+1} \\
& =\Lambda \sum_{n=0}^{\infty} \sum_{r=0}^{n+1}\left(\begin{array}{c}
n+1 \\
r
\end{array}\right) \frac{\left(\sum_{j=1}^{k} \theta_{j} t^{j}\right)^{n+1-r} \alpha^{r}}{(n+1)},
\end{aligned}
$$

by binomial theorem. Now, on splitting the inner summation of (2.7) we obtain

$$
H(t)=\Lambda \sum_{n=0}^{\infty} \sum_{r=0}^{n} \frac{\left(\begin{array}{c}
n+1 \\
r
\end{array}\right)}{(n+1)}\left(\sum_{j=1}^{k} \theta_{j} t^{j}\right)^{n+1-r} \alpha^{r}+\Lambda \sum_{n=0}^{\infty} \frac{\alpha^{n+1}}{(n+1)} .
$$

By applying (1.4) in the first term and since the second term is the logarithmic expansion of $[-\ln (1-\alpha)]$, we have the following from (2.8).

$$
H(t)=\Lambda \sum_{n=0}^{\infty} \sum_{r=0}^{\infty} \frac{\left(\begin{array}{c}
n+r+1 \\
r
\end{array}\right)}{(n+r+1)}\left(\sum_{j=1}^{k} \theta_{j} t^{j}\right)^{n+1} \alpha^{r}-\Lambda \ln (1-\alpha) .
$$

Now, by applying the multinomial expansion in (2.9), we have

$$
H(t)=\Lambda \sum_{n=0}^{\infty} \sum_{r=0}^{\infty} \frac{\left(\begin{array}{c}
n+r+1 \\
r
\end{array}\right)}{(n+r+1)} \sum_{J_{n}} \frac{(n+1) !}{y_{1} ! y_{2} ! \ldots y_{k} !} \theta_{1}^{y_{1}} \theta_{2}^{y_{2}} \ldots \theta_{k}^{y_{k}} \alpha^{r} t^{\delta}-\Lambda \ln (1-\alpha),
$$

in which $\delta=\sum_{j=1}^{k} j y_{j}, \sum_{J_{n}}$ denote the summation over all $k$ tuples $\left(y_{1}, y_{2}, \ldots, y_{k}\right)$ of non-negative integers in the set $J_{n}=\left\{\left(y_{1}, y_{2}, \ldots, y_{k}\right): \sum_{j=1}^{k} y_{j}=n+1\right\}$. On rearranging the terms, we have

$$
H(t)=\Lambda \sum_{n=0}^{\infty} \sum_{r=0}^{\infty} \frac{(n+r+1) !}{(n+1) !(n+r+1)} \frac{\alpha^{r}}{r !} \sum_{J_{n}} \frac{(n+1) !}{y_{1} ! y_{2} ! \ldots y_{k} !} \theta_{1}^{y_{1}} \theta_{2}^{y_{2}} \ldots \theta_{k}^{y_{k}} t^{\delta}-\Lambda \ln (1-\alpha)
$$

which implies the following, in the light of the relation $(1+v)_{p}=\frac{(v+p) !}{v !}$ and the definition of the Gauss hypergeometric function.

$$
\begin{aligned}
H(t) & =\Lambda \sum_{n=0}^{\infty} \sum_{r=0}^{\infty} \frac{(n+1)_{r}}{(n+1)} \frac{\alpha^{r}}{r !} \sum_{J_{n}} \frac{(n+1) !}{y_{1} ! y_{2} ! \ldots y_{k} !} \theta_{1}^{y_{1}} \theta_{2}^{y_{2}} \ldots \theta_{k}^{y_{k}} t^{\delta}-\Lambda \ln (1-\alpha) \\
& =\Lambda \sum_{n=0}^{\infty} \sum_{J_{n}}(1-\alpha)^{-(n+1)} \frac{n !}{y_{1} ! y_{2} ! \ldots y_{k} !} \theta_{1}^{y_{1}} \theta_{2}^{y_{2}} \ldots \theta_{k}^{y_{k}} t^{\delta}-\Lambda \ln (1-\alpha),
\end{aligned}
$$


in the light of (1.5). Now, on equating the coefficients of $t^{y}$ on the right hand side expressions of (2.5) and (2.13) we get (2.4).

Next we obtain the mean and variance of the $\operatorname{ALSD}(k)$ through following theorem.

Theorem 2.2 The mean and variance of the $\operatorname{ALSD}(k)$ are the following, in which $\delta=\left(1-\sum_{j=1}^{k} \theta_{j}-\alpha\right)^{-1}$ and $\lambda=\sum_{j=1}^{k} j \theta_{j}$

$$
\text { Mean }=\Lambda \delta \lambda
$$

and

$$
\text { Variance }=\Lambda \delta\left[\sum_{j=1}^{k} j(j-1) \theta_{j}+\lambda+\Lambda \delta \lambda^{2}\right]
$$

Proof follows from the fact that

$$
\text { Mean }=H^{(1)}(1)
$$

and

$$
\text { Variance }=H^{(2)}(1)+H^{(1)}(1)-\left[H^{(1)}(1)\right]^{2},
$$

where for $r=1,2, \ldots$

$$
H^{(r)}(t)=\frac{d^{r} H(t)}{d t^{r}} / t=1
$$

\section{Maximum Likelihood Estimation}

In this section we discuss the estimation of the parameters of the $\operatorname{ALSD}(k)$ by the method of maximum likelihood estimation.

Let $a(y)$ be the observed frequency of $y$ events and let $z$ be the highest value of $y$ observed. Then the likelihood function $L$ of the sample is the following, in which $q(y)$ is the pmf of the $\operatorname{ALSD}(k)$ as given in (2.4).

$$
L=\prod_{y=0}^{z}[q(y)]^{a(y)}=[q(0)]^{a(0)} \prod_{y=1}^{z}[q(y)]^{a(y)}
$$

Taking logarithm on both sides of (3.1), we have

$$
\begin{aligned}
\ln L & =a(0) \ln [q(0)]+\sum_{y=1}^{z} a(y) \ln [q(y)] \\
& =a(0) \ln [q(0)]+\sum_{y=1}^{z} a(y)\left[\ln \Lambda+\ln \phi\left(y ; \theta_{1}, \theta_{2}, \ldots, \theta_{k}, \alpha\right)\right],
\end{aligned}
$$

where $\Lambda$ is as given in (2.4) and

$$
\phi\left(y ; \theta_{1}, \theta_{2}, \ldots, \theta_{k}, \alpha\right)=\sum_{J_{y}} \frac{n !}{(1-\alpha)^{(1+y)}} \frac{\theta_{1}^{y_{1}}}{y_{1} !} \frac{\theta_{2}^{y_{2}}}{y_{2} !} \cdots \frac{\theta_{k}^{y_{k}}}{y_{k} !} .
$$


where $\sum_{J_{y}}$ is as defined in (2.4). Let $\hat{\theta}_{j}$ denote the maximum likelihood estimator of the parameter $\theta_{j}$ for $j=1,2, \ldots, k$ and $\hat{\alpha}$ denote the maximum likelihood estimator of the parameter $\alpha$ of the $\operatorname{ALSD}(k)$. On differentiating (3.2) partially with respect to the parameters $\theta_{j}$, for $j=1,2, \ldots, k$ and $\alpha$ and equating to zero, we get the following system of likelihood equations.

$$
-a(0) \Lambda \delta+\sum_{y=1}^{z} a(y)\left[\frac{\sum_{J_{y}} \frac{n !}{(1-\alpha)^{(1+y)}} \frac{\theta_{1}^{y_{1}}}{y_{1} !} \frac{\theta_{2}^{y_{2}}}{y_{2} !} \cdots \frac{\theta_{j-1}^{y_{j-1}}}{y_{j-1} !} \frac{\theta_{j}^{y_{j}-1}}{\left(y_{j}-1\right) !} \frac{\theta_{j+1}^{y_{j+1}}}{y_{j+1} !} \cdots \frac{\theta_{k}^{y_{k}}}{y_{k} !}}{\phi\left(y ; \theta_{1}, \theta_{2}, \ldots, \theta_{k}, \alpha\right)}\right]=0
$$

and

$$
a(0)\left[\frac{1}{-(1-\alpha) \ln (1-\alpha)}-\Lambda \delta\right]+\sum_{y=1}^{z} a(y)\left[\frac{\sum_{y} \frac{n !(y+1)}{(1-\alpha)^{y}} \frac{\theta_{1}^{y_{1}}}{y_{1} !} \frac{\theta_{2}^{y_{2}}}{y_{2} !} \cdots \frac{\theta_{k}^{y_{k}}}{y_{k} !}}{\phi\left(y ; \theta_{1}, \theta_{2}, \ldots, \theta_{k}, \alpha\right)}\right]=0
$$

Now, on solving the likelihood equations given in (3.3) and (3.4) by using mathematical softwares such as MATLAB, MATHCAD, MATHEMATICA etc., we can obtain the maximum likelihood estimators of the parameters of the $\operatorname{ALSD}(k)$. When the likelihood equations do not always have a solution, the maximum of the likelihood function attained at the border of the domain of parameters. So, we obtained the second order partial derivatives of $\ln [q(y)]$ with respect to parameters $\theta_{j}$, for $j=1,2, \ldots, k$ and $\alpha$, and by using MATHCAD software we observed that these equations give negative values for all $\theta_{j}>0$ and $\alpha \geq 0$ such that $\alpha+\sum_{j=1}^{k} \theta_{j}<1$. Thus, the density of the $\operatorname{ALSD}(k)$ is log-concave and hence the maximum likelihood estimators of the parameters $\theta_{j}$ and $\alpha$ are unique under these parametric restrictions (cf. Puig, 23). In practice, one can estimate the parameters $\theta_{j}$ for $j=1,2, \ldots, k$ and $\alpha$ of the $\operatorname{ALSD}(k)$ for particular values of $k$, say $k=1,2, \ldots$.

\section{Applications}

For numerical applications, here we consider two real life data sets of which the first data set is the observed distribution of bacterial clumps per field in a milk film taken from Bliss and Fisher [1] and the second data set is the experimental evidence concerning contagious distributions in ecology taken from Evans [3]. We have fitted the LWZD, the ALSD and the $\operatorname{ALSD}(k)$ to these data sets and the results thus obtained along with the corresponding values of the expected frequencies, Chi-square statistic, degrees of freedom (d.f.), P, Akaike information criterion (AIC), Bayesian information criterion (BIC) and the second order Akaike information criterion (AICc) for which the $\operatorname{ALSD}(k)$ gives the better fit for each of these models are presented in the Tables 1 and 2. Based on the values of Chi-square statistic, P, AIC, BIC and AICc from these tables we can be observe that the $\operatorname{ALSD}(k)$ gives a better fit to the first data set for $k=3$ and the second data set for $k=2$, compared to the existing models- the LWZD and the ALSD. 
The alpha-logarithmic series distribution of order $k$

Table 1. Observed frequencies and expected frequencies of the LWZD, the ALSD, and the ALSD $(k)$ by the method of maximum likelihood for the first data set.

\begin{tabular}{|c|c|c|c|c|c|c|}
\hline \multirow{2}{*}{$\begin{array}{l}\text { No. per } \\
\text { unit }\end{array}$} & \multirow{2}{*}{$\begin{array}{l}\text { Observed } \\
\text { frequency }\end{array}$} & \multirow[t]{2}{*}{ LWZD } & \multirow[t]{2}{*}{ ALSD } & \multicolumn{3}{|c|}{$\operatorname{ALSD}(k)$} \\
\hline & & & & $k=2$ & $k=3$ & $k=4$ \\
\hline 0 & 56 & 64.00 & 69.41 & 60.66 & 53.01 & 53.65 \\
\hline 1 & 104 & 126.98 & 118.56 & 114.01 & 103.07 & 94.14 \\
\hline 2 & 80 & 57.78 & 69.85 & 70.37 & 85.05 & 74.66 \\
\hline 3 & 62 & 35.05 & 61.64 & 64.56 & 60.25 & 57.02 \\
\hline 4 & 42 & 23.92 & 32.52 & 40.52 & 43.45 & 50.92 \\
\hline 5 & 27 & 17.41 & 18.56 & 21.06 & 30.14 & 23.58 \\
\hline 6 & 9 & 13.20 & 11.01 & 9.03 & 8.01 & 15.45 \\
\hline 7 & 9 & 10.30 & 8.16 & 8.01 & 7.75 & 12.65 \\
\hline 8 & 5 & 8.20 & 5.17 & 5.03 & 5.04 & 7.75 \\
\hline 9 & 3 & 6.64 & 3.69 & 2.01 & 3.12 & 4.91 \\
\hline 10 & 3 & 36.52 & 1.43 & 4.74 & 1.11 & 5.27 \\
\hline Total & 400 & 400 & 400 & 400 & 400 & 400 \\
\hline $\begin{array}{c}\text { Estimates } \\
\text { of the parameters }\end{array}$ & & $\begin{array}{l}\hat{\theta}=0.91 \\
\hat{\omega}=0.16\end{array}$ & $\begin{array}{l}\hat{\theta}=0.51 \\
\hat{\alpha}=0.42\end{array}$ & $\begin{array}{l}\hat{\theta}_{1}=0.41 \\
\hat{\theta}_{2}=0.25 \\
\hat{\alpha}=0.19\end{array}$ & $\begin{array}{l}\hat{\theta}_{1}=0.35 \\
\hat{\theta}_{2}=0.21 \\
\hat{\theta}_{3}=0.11 \\
\hat{\alpha}=0.15\end{array}$ & $\begin{array}{l}\hat{\theta}_{1}=0.36 \\
\hat{\theta}_{2}=0.18 \\
\hat{\theta}_{3}=0.09 \\
\hat{\theta}_{4}=0.01 \\
\hat{\alpha}=0.16\end{array}$ \\
\hline Chi-square value & & 88.89 & 13.79 & 6.59 & 2.13 & 10.45 \\
\hline d.f. & & 8 & 7 & 6 & 4 & 4 \\
\hline P-value & & $<0.0001$ & 0.05 & 0.36 & 0.77 & 0.03 \\
\hline AIC & & 3942.50 & 3352.34 & 3354.34 & 3348.32 & 3350.32 \\
\hline $\mathrm{BIC}$ & & 3950.48 & 3360.32 & 3359.98 & 3350.78 & 3353.33 \\
\hline $\mathrm{AICc}$ & & 3942.53 & 3352.37 & 3354.06 & 3348.42 & 3350.50 \\
\hline
\end{tabular}


Table 2. Observed frequencies and expected frequencies of the LWZD, the ALSD, and the $\operatorname{ALSD}(k)$ by the method of maximum likelihood for the second data set.

\begin{tabular}{|c|c|c|c|c|c|}
\hline \multirow{2}{*}{$\begin{array}{l}\text { No. per } \\
\text { unit }\end{array}$} & \multirow{2}{*}{$\begin{array}{l}\text { Observed } \\
\text { frequency }\end{array}$} & \multirow[t]{2}{*}{ LWZD } & \multirow[t]{2}{*}{ ALSD } & \multicolumn{2}{|c|}{$\operatorname{ALSD}(k)$} \\
\hline & & & & $k=2$ & $k=3$ \\
\hline 0 & 12 & 15.00 & 17.56 & 10.38 & 6.01 \\
\hline 1 & 22 & 26.95 & 32.04 & 21.35 & 26.53 \\
\hline 2 & 19 & 12.80 & 14.44 & 20.50 & 24.62 \\
\hline 3 & 17 & 8.10 & 8.68 & 15.12 & 15.19 \\
\hline 4 & 15 & 5.78 & 5.87 & 14.40 & 12.80 \\
\hline 5 & 6 & 4.39 & 4.24 & 8.01 & 7.01 \\
\hline 6 & 5 & 3.48 & 3.18 & 5.05 & 4.05 \\
\hline 7 & 2 & 2.83 & 2.45 & 3.20 & 1.42 \\
\hline 8 & 2 & 20.67 & 11.54 & 1.99 & 1.97 \\
\hline Total & 100 & 100 & 100 & 100 & 100 \\
\hline $\begin{array}{c}\text { Estimates } \\
\text { of the parameters }\end{array}$ & & $\begin{array}{l}\hat{\theta}=0.95 \\
\hat{\omega}=0.15\end{array}$ & $\begin{aligned} \hat{\theta} & =0.55 \\
\hat{\alpha} & =0.39\end{aligned}$ & $\begin{array}{l}\hat{\theta}_{1}=0.62 \\
\hat{\theta}_{2}=0.35 \\
\hat{\alpha}=0.09\end{array}$ & $\begin{array}{c}\hat{\theta}_{1}=0.52 \\
\hat{\theta}_{2}=0.34 \\
\hat{\theta}_{3}=0.01 \\
\hat{\alpha}=0.11\end{array}$ \\
\hline Chi-square value & & 46.36 & 37.40 & 1.42 & 7.99 \\
\hline d.f. & & 4 & 4 & 4 & 2 \\
\hline $\mathrm{P}$-value & & $<0.0001$ & $<0.0001$ & 0.84 & 0.02 \\
\hline $\mathrm{AIC}$ & & 889.98 & 837.22 & 833.92 & 836.92 \\
\hline $\mathrm{BIC}$ & & 890.56 & 838.02 & 834.91 & 837.45 \\
\hline $\mathrm{AICc}$ & & 890.10 & 837.34 & 834.17 & 837.34 \\
\hline
\end{tabular}

\section{Testing of the Hypothesis}

In this section we discuss the testing of the hypothesis $H_{0}: \theta_{i_{1}}=\theta_{i_{2}}=\cdots=\theta_{i_{m}}=0$, for any particular subset $\left\{i_{1}, i_{2}, \ldots, i_{m}\right\}$ of the set $\{1,2, \ldots, k\}$, by using generalized likelihood ratio test and Rao's efficient score test.

\subsection{Generalized Likelihood Ratio Test}

In case of generalized likelihood ratio test, the test statistic is

$$
-2 \ln \lambda=2\left(l_{1}-l_{2}\right),
$$

where $l_{1}=\ln L(\underline{\hat{\theta}} ; x)$, in which $\underline{\hat{\theta}}$ is the maximum likelihood estimator of $\underline{\theta}=\left(\theta_{1}, \theta_{2}, \ldots, \theta_{k} ; \alpha\right)$ with no restrictions and $l_{2}=\ln L\left(\underline{\hat{\theta}}^{*} ; x\right)$, in which $\underline{\hat{\theta}}^{*}$ is the maximum likelihood estimator of $\underline{\theta}$ under $H_{0}$. The log- 
likelihood function $\ln L=\ln L(\underline{\theta}, \underline{x})$ is as defined in (3.2) and the test statistic given in (5.1) is asymptotically distributed as chi-square with $m$ degrees of freedom (for details see Rao, 24).

For testing the significance of the additional parameters of the $\operatorname{ALSD}(k)$ for $k=3$ in case of first data set we consider the following three tests:

Test 1: $H_{0}^{(1)}: \theta_{2}=0$ against $H_{1}^{(1)}: \theta_{2} \neq 0$

Test 2: $H_{0}^{(2)}: \theta_{3}=0$ against $H_{1}^{(2)}: \theta_{3} \neq 0$

Test 3: $H_{0}^{(3)}: \theta_{2}=\theta_{3}=0$ against $H_{1}^{(3)}: \theta_{2} \neq 0, \theta_{3} \neq 0$.

In case of the second data set we consider the null hypothesis as $H_{0}^{(1)}: \theta_{2}=0$ against alternative hypothesis $H_{1}^{(1)}: \theta_{2} \neq 0$ for testing the significance of the additional parameter $\theta_{2}$ of the $\operatorname{ALSD}(k)$ for $k=2$. We have computed the values of $\ln L(\underline{\hat{\theta}} ; x), \ln L\left(\underline{\hat{\theta}}^{*} ; x\right)$ and the test statistic given in (5.1) in all the above situations and presented in Table 3 .

Table 3. Computed values of $\ln L(\underline{\hat{\theta}} ; x), \ln L\left(\underline{\hat{\theta}}^{*} ; x\right)$ and the generalized likelihood ratio test statistic.

\begin{tabular}{lllllll}
\hline & & $\ln L\left(\underline{\hat{\theta}}^{*} ; x\right)$ & $\ln L(\underline{\hat{\theta}} ; x)$ & $\begin{array}{l}\text { Test } \\
\text { statistic }\end{array}$ & d.f & \multicolumn{2}{c}{$\begin{array}{l}\text { Chi-square } \\
\text { value (tabled value) }\end{array}$} \\
\hline Data set 1 & Test 1 & -1676.71 & -1674.17 & 6.84 & 1 & 3.84 \\
\cline { 2 - 7 } & Test 2 & -1677.01 & -1674.17 & 5.68 & 1 & 3.84 \\
\cline { 2 - 7 } & Test 3 & -1678.25 & -1674.17 & 8.16 & 2 & 5.99 \\
\hline Data set 2 & & -420.26 & -417.46 & 4.56 & 1 & 3.84 \\
\hline
\end{tabular}

From table 3, it can be observed that the calculated value of the test statistic is greater than the tabled value in all the above situation and hence one can conclude that of the additional parameters $\theta_{2}$ and $\theta_{3}$ are significant in case first data set and the additional parameter $\theta_{2}$ is significant in case of second data set, at $5 \%$ level of significance.

\subsection{Rao's Efficient Score Test}

Here the test statistic is

$$
S=M^{\prime} \phi^{-1} M
$$

in which

$$
M^{\prime}=\left(\frac{1}{\sqrt{n}} \frac{\partial \log L}{\partial \theta_{1}}, \frac{1}{\sqrt{n}} \frac{\partial \log L}{\partial \theta_{2}}, \cdots, \frac{1}{\sqrt{n}} \frac{\partial \log L}{\partial \theta_{k}}, \frac{1}{\sqrt{n}} \frac{\partial \log L}{\partial \alpha}\right)
$$

and $\phi$ is the Fisher information matrix. The test statistic $S$ given in (5.2) follows chi-square distribution with $m$ degrees of freedom (for details see Rao, 24).

For testing the significance of the additional parameters of the $\operatorname{ALSD}(k)$ for $k=3$ in case of the first data set we consider the following three tests: 
Test 1: $H_{0}^{(1)}: \theta_{2}=0$ against $H_{1}^{(1)}: \theta_{2} \neq 0$

Test 2: $H_{0}^{(2)}: \theta_{3}=0$ against $H_{1}^{(2)}: \theta_{3} \neq 0$

Test 3: $H_{0}^{(3)}: \theta_{2}=\theta_{3}=0$ against $H_{1}^{(3)}: \theta_{2} \neq 0, \theta_{3} \neq 0$.

For testing of the significance of the additional parameter $\theta_{2}$ of the $\operatorname{ALSD}(k)$ for $k=2$ in case of the second data set we consider the null hypothesis as $H_{0}^{(1)}: \theta_{2}=0$ against the alternative hypothesis $H_{1}^{(1)}: \theta_{2} \neq 0$. We have computed the values of $S$ for the $\operatorname{ALSD}(k)$ in Test 1 as $S_{1}$, in Test 2 as $S_{2}$, in Test 3 as $S_{3}$ of the $\operatorname{ALSD}(k)$ in the case of first data set and $S_{4}$ as in the case of second data set and presented them in Table 4 . The computational details of $S_{i}$ for $i=1,2,3$ and 4 are given in Appendix A.

Table 4. Computed values of Rao's efficient score test statistic.

\begin{tabular}{lllll}
\hline & & Test statistic & d.f & $\begin{array}{l}\text { Chi-square value } \\
\text { (tabled value) }\end{array}$ \\
\hline Data set 1 & $S_{1}$ & 4.11 & 1 & 3.84 \\
\cline { 2 - 5 } & $S_{2}$ & 4.12 & 1 & 3.84 \\
\cline { 2 - 5 } & $S_{3}$ & 29.41 & 2 & 5.991 \\
\hline Data set 2 & $S_{4}$ & 11.62 & 1 & 3.84 \\
\hline
\end{tabular}

From table 4, it can be observed that the calculated value of the test statistic is greater than the tabled value in all the above situations and hence one can conclude that the additional parameters $\theta_{2}$ and $\theta_{3}$ are significant in case first data set and the additional parameter $\theta_{2}$ is significant in case of the second data set, at $5 \%$ level of significance.

\section{Simulation}

It is quite difficult to assess the theoretical performance of the estimators of different parameters of the $\operatorname{ALSD}(k)$ obtained by the method of maximum likelihood. So in this section we have attempted a simulation study for comparing the performance of the estimators. We have simulated three data sets of sample sizes 150,300 and 600 in case of both the over-dispersed and under-dispersed situations of the $\operatorname{ALSD}(k)$ for $k=2,3$ and 4, by using Markov Chain Monte Carlo (MCMC) simulation procedure, and considered 200 replications in each case. The initial value of the parameters assumed for simulating the data sets according to the nature of dispersion and the computed the values of bias and standard errors in case of each estimators are summarized in Tables 5, 6 and 7. From these tables it can be observed that both the bias and standard errors of the estimators of the parameters are in decreasing order as the sample size increases. 
Table 5. Bias and standard error (within parenthesis) of the estimators of the parameters $\theta_{1}, \theta_{2}$ and $\alpha$ of the $\operatorname{ALSD}(k)$ for $k=2$ corresponding to the parameter set: for (i) $\theta_{1}=0.4505, \theta_{2}=0.3504, \alpha=0.1542$ (over-dispersion) and (ii) $\theta_{1}=0.3501, \theta_{2}=0.0541, \alpha=0.1014$ (under-dispersion).

\begin{tabular}{|c|c|c|c|c|}
\hline Parameter set & Sample size & \multicolumn{3}{|c|}{ Maximum likelihood estimation } \\
\cline { 2 - 5 } & & $\hat{\theta}_{1}$ & $\hat{\theta}_{2}$ & $\hat{\alpha}$ \\
\hline \multirow{3}{*}{ (i) } & 150 & 0.0704 & 0.0943 & 0.0671 \\
& & $(0.1082)$ & $(0.1068)$ & $(0.1149)$ \\
\cline { 2 - 5 } & 300 & 0.0514 & 0.0663 & 0.0548 \\
& & $(0.0995)$ & $(0.0894)$ & $(0.0917)$ \\
\cline { 2 - 5 } & 600 & 0.0403 & 0.0399 & 0.0421 \\
& & $(0.0894)$ & $(0.0819)$ & $(0.0843)$ \\
\hline \multirow{6}{*}{ (ii) } & 150 & 0.0696 & 0.0439 & 0.0527 \\
& & $(0.1578)$ & $(0.1230)$ & $(0.1549)$ \\
\cline { 2 - 5 } & 300 & 0.0507 & 0.0331 & 0.0343 \\
& & $(0.1334)$ & $(0.1109)$ & $(0.1196)$ \\
\cline { 2 - 5 } & & 0.0373 & 0.0226 & 0.0123 \\
& 600 & $(0.1221)$ & $(0.1068)$ & $(0.1091)$ \\
\hline
\end{tabular}

Table 6. Bias and standard errors (within parenthesis) of the estimators of the parameters $\theta_{1}, \theta_{2}, \theta_{3}$ and $\alpha$ of the $\operatorname{ALSD}(k)$ for $k=3$ corresponding to the parameter set: (i) $\theta_{1}=0.3608, \theta_{2}=0.2691, \theta_{3}=0.1451, \alpha=0.1614$ (over-dispersion) and (ii) $\theta_{1}=0.7208$, $\theta_{2}=0.0591, \theta_{3}=0.0301, \alpha=0.0528$ (under-dispersion).

\begin{tabular}{|c|c|c|c|c|c|}
\hline \multirow[t]{2}{*}{ Parameter set } & \multirow[t]{2}{*}{ Sample size } & \multicolumn{4}{|c|}{ Maximum likelihood estimation } \\
\hline & & $\hat{\theta}_{1}$ & $\hat{\theta}_{2}$ & $\hat{\theta}_{3}$ & $\hat{\alpha}$ \\
\hline \multirow{3}{*}{ (i) } & 150 & $\begin{array}{c}0.0717 \\
(0.1187)\end{array}$ & $\begin{array}{c}0.0522 \\
(0.1386)\end{array}$ & $\begin{array}{c}0.0686 \\
(0.1273)\end{array}$ & $\begin{array}{c}0.1053 \\
(0.1183)\end{array}$ \\
\hline & 300 & $\begin{array}{c}0.0609 \\
(0.1077)\end{array}$ & $\begin{array}{c}0.0460 \\
(0.1058)\end{array}$ & $\begin{array}{c}0.0499 \\
(0.1105)\end{array}$ & $\begin{array}{c}0.0873 \\
(0.0975)\end{array}$ \\
\hline & 600 & $\begin{array}{c}0.0487 \\
(0.0985) \\
\end{array}$ & $\begin{array}{c}0.0389 \\
(0.0917) \\
\end{array}$ & $\begin{array}{c}0.0289 \\
(0.0959) \\
\end{array}$ & $\begin{array}{c}0.0713 \\
(0.0854) \\
\end{array}$ \\
\hline \multirow{3}{*}{ (ii) } & 150 & $\begin{array}{c}0.1077 \\
(0.1183)\end{array}$ & $\begin{array}{c}0.0489 \\
(0.1005)\end{array}$ & $\begin{array}{c}0.0210 \\
(0.1068)\end{array}$ & $\begin{array}{c}0.0443 \\
(0.1039)\end{array}$ \\
\hline & 300 & $\begin{array}{c}0.0868 \\
(0.1077)\end{array}$ & $\begin{array}{c}0.0376 \\
(0.0935)\end{array}$ & $\begin{array}{c}0.0199 \\
(0.0877)\end{array}$ & $\begin{array}{c}0.0416 \\
(0.0819)\end{array}$ \\
\hline & 600 & $\begin{array}{c}0.0667 \\
(0.0970)\end{array}$ & $\begin{array}{c}0.0332 \\
(0.0872)\end{array}$ & $\begin{array}{c}0.0149 \\
(0.0748)\end{array}$ & $\begin{array}{c}0.0174 \\
(0.0755)\end{array}$ \\
\hline
\end{tabular}


Table 7. Bias and standard errors (within parenthesis) of the estimators of the parameters $\theta_{1}, \theta_{2}$, $\theta_{3}, \theta_{4}$ and $\alpha$ of the ALSD ( $k$ ) for $k=4$ corresponding to the parameters set: (i) $\theta_{1}=0.4123$, $\theta_{2}=0.2304, \theta_{3}=0.1532, \theta_{4}=0.1005, \alpha=0.0989$ (over-dispersion) and (ii) $\theta_{1}=0.7825$, $\theta_{2}=0.0451, \theta_{3}=0.0326, \theta_{4}=0.0578, \alpha=0.0785$ (under-dispersion).

\begin{tabular}{|c|l|l|l|l|l|l|}
\hline \multirow{2}{*}{$\begin{array}{c}\text { Parameter } \\
\text { set }\end{array}$} & $\begin{array}{l}\text { Sample } \\
\text { size }\end{array}$ & \multicolumn{5}{|c|}{ Maximum likelihood estimation: } \\
\cline { 2 - 7 } & & $\hat{\theta}_{1}$ & $\hat{\theta}_{2}$ & \multicolumn{1}{|c|}{$\hat{\theta}_{3}$} & \multicolumn{1}{c|}{$\hat{\theta}_{4}$} & $\hat{\alpha}$ \\
\hline \multirow{4}{*}{ (i) } & 150 & 0.1011 & 0.0708 & 0.0934 & 0.0544 & 0.0781 \\
& & $(0.1025)$ & $(0.1034)$ & $(0.1010)$ & $(0.0872)$ & $(0.0794)$ \\
\cline { 2 - 7 } & 300 & 0.0561 & 0.0503 & 0.0571 & 0.0384 & 0.0527 \\
& & $(0.0970)$ & $(0.0906)$ & $(0.0854)$ & $(0.0806)$ & $(0.0663)$ \\
\cline { 2 - 7 } & 600 & 0.0322 & 0.0397 & 0.0268 & 0.0104 & 0.0428 \\
& & $(0.0877)$ & $(0.0806)$ & $(0.0768)$ & $(0.0728)$ & $(0.0608)$ \\
\hline \multirow{5}{*}{ (ii) } & 150 & 0.1168 & 0.0416 & 0.0235 & 0.0481 & 0.0662 \\
& & $(0.0854)$ & $(0.0787)$ & $(0.0755)$ & $(0.0721)$ & $(0.0707)$ \\
\cline { 2 - 7 } & 300 & 0.0874 & 0.0349 & 0.0197 & 0.0311 & 0.0439 \\
& & $(0.0731)$ & $(0.0686)$ & $(0.0678)$ & $(0.0648)$ & $(0.0624)$ \\
\cline { 2 - 7 } & 600 & 0.0474 & 0.0249 & 0.0029 & 0.0122 & 0.0223 \\
& & $(0.0629)$ & $(0.0529)$ & $(0.0532)$ & $(0.0583)$ & $(0.566)$ \\
\hline
\end{tabular}

\section{Appendix. A}

We have computed the values of the Rao's efficient score test statistic corresponding to the tests described in section 5.2 as follows:

$$
\begin{aligned}
& S_{1}=\left(\begin{array}{llll}
0.77 & 1.78 & 5.26 & 7.76
\end{array}\right)\left[\begin{array}{ccccc}
0.09 & 0.08 & -0.18 & 0.01 \\
0.08 & 0.18 & -0.28 & 0.03 \\
-0.18 & -0.28 & 0.51 & -0.07 \\
0.01 & 0.03 & -0.07 & 0.03
\end{array}\right]\left(\begin{array}{l}
0.77 \\
1.78 \\
5.26 \\
7.76
\end{array}\right)=4.11 \\
& S_{2}=\left(\begin{array}{lllll}
0.06 & 4.26 & 9.26 & 11.26
\end{array}\right)\left[\begin{array}{cccc}
0.07 & -0.05 & -0.07 & 0.05 \\
0.05 & 0.09 & 0.03 & -0.08 \\
-0.07 & 0.03 & 0.12 & -0.09 \\
0.05 & -0.08 & -0.09 & 0.13
\end{array}\right]\left(\begin{array}{c}
0.06 \\
4.26 \\
9.26 \\
11.26
\end{array}\right)=4.12 \\
& S_{3}=\left(\begin{array}{llllll}
0.58 & 6.28 & 10.96 & 12.75
\end{array}\right)\left[\begin{array}{cccc}
0.18 & 0.01 & -0.01 & -0.18 \\
0.01 & 0.07 & -0.03 & -0.04 \\
-0.01 & -0.03 & 0.09 & -0.05 \\
-0.18 & -0.04 & -0.05 & 0.27
\end{array}\right]\left(\begin{array}{c}
0.58 \\
6.28 \\
10.96 \\
12.75
\end{array}\right)=29.41 \\
& S_{4}=\left(\begin{array}{llll}
1.56 & 3.56 & 8.53
\end{array}\right)\left[\begin{array}{ccc}
0.15 \\
0.09 \\
-0.22 & 0.09 & -0.22 \\
0.09 & -0.16 \\
-0.16 & 0.34
\end{array}\right]\left(\begin{array}{c}
1.56 \\
3.56 \\
8.53
\end{array}\right)=11.62
\end{aligned}
$$




\section{References}

[1] Bliss, C. I. and Fisher, R. A. (1953). Fitting the negative binomial distribution to biological data. Biometrics, 9, 176-200.

[2] Chatfield, C., Ehrenberg, A. S. C. and Goodhardt, G. J. (1966). Progress on a simplified model of stationary purchasing behavior (with discussion). Journal of the Royal Statistical Society, Series A, 129, 317-367.

[3] Evans, D. A. (1953). Experimental evidence concerning contagious distribution in ecology. Biometrika, 40, 186-211.

[4] Fisher, R. A., Corbet, A. S. and Williams, C. B. (1943). The relation between the number of species and the number of individuals in a random sample of an animal population. Journal of Animal Ecology, 12, $42-58$.

[5] Jain, G. C. and Gupta, R. P. (1973). A logarithmic series type distribution. Trabaios de Estadistica, 24, 99-105.

[6] Johnson, N. L., Kemp, A.W. and Kotz, S. (2005). Univariate Discrete Distributions. Wiley, New York.

[7] Kempton, R. A. (1975). A generalized form Fisher's logarithmic series. Biometrika, 62, 29-38.

[8] Khang, T. F. and Ong, S. H. (2007). A new generalization of the logarithmic distribution arising from the inverse trinomial distribution. Communication in Statistics-Theory and Methods, 36, 3-21.

[9] Khatri, C. G. (1961). On the distribution obtained by varying the number of trails in a binomial distribution. Annals of the Institute of Statistical Mathematics, 13, 47-51.

[10] Kumar, C. S. (2009). A class of discrete distributions of order k. Journal of Statistical Theory and Practice, 3,795-803.

[11] Kumar, C. S. (2010). Binomial Poisson distribution revisited. Economic Quality Control, 25, 183-188.

[12] Kumar, C. S. and Nair, B. U. (2013).On suttering hyper - Poisson and its properties. Sri Lankan Journal of Applied Statistics, $14,41-54$.

[13] Kumar, C. S. and Nair, B. U. (2013a). Order k version of the alternative hyper-Poisson distribution. Economic Quality Control.28, 97-104.

[14] Kumar, C. S. and Riyaz, A. (2013). On estimating the parameters of an extended logarithmic series distribution and its applications. Communications for Statistical Applications and Methods, 20, 417-425.

[15] Kumar, C. S. and Riyaz, A. (2013a). A modified version of logarithmic series distribution and its applications. Communication in Statistics-Theory and Methods, (accepted for publication).

[16] Kumar, C. S. and Riyaz, A. (2014). An alternative to 'the logarithmic-with-zeros distribution' and its generalization: Properties and estimation. South African Statistical Journal, 48, 253-266.

[17] Kumar, C. S. and Riyaz, A. (2014a). Logarithmic series distribution of order k. The Aligarh Journal of Statistics, 34, 45-54.

[18] Kumar, C. S. and Shibu, D. S. (2013). On intervened stuttering Poisson distribution and its application. Journal of Statistical Theory and Practice, 7, 544-557.

[19] Mathai, A. M. and Haubold, H. J. (2008). Special Functions for Applied Scientists. Springer, New York.

[20] Ong, S. H. (2000). On a generalization of the log-series distribution. Journal of Applied Statistical Science, 10(1), $77-88$.

[21] Panaretos, J. and Xekalaki, E. (1986). On some distributions arising from certain generalized sampling schemes. Communications in Statistics-Theory and Methods, 15, 873-891.

[22] Philippou, A. N. (1984). The negative binomial distribution of order k and some of its properties. Biometrical Journal, 26, 789794.

[23] Puig, P. (2003). Characterizing additively closed discrete models by a property of their MLEs with an application to generalized Hermite distribution. Journal of American Statistical Association, 98, 687-692.

[24] Rao, C. R. (1973). Linear Statistical Inference and its Application. John Wiley, New York.

[25] Slater, L. J. (1966). Generalized Hypergeometric Functions. Cambridge University Press, Cambridge.

[26] Tripathi, R. C. and Gupta, R. C. (1985). A generalization of the log-series distribution. Communication in Statistics-Theory and Methods, 14, 1779-1799.

[27] Tripathi, R. C. and Gupta, R. C. (1988). Another generalization of the logarithmic series and the geometric distribution. Communication in Statistics-Theory and Methods, 17, 1541-1547.

[28] Williams, C. B. (1947). The logarithmic series and its application to biological problems. Journal of Ecology, 34, $253-272$.

[29] Williams, C. B. (1964). Patterns in the Balance of Nature. London, Academic Press.

[30] Williamson, E. and Bretherton (1964). Tables of the logarithmic series distribution. Annals of Mathematical Statistics, 35, 384297. 